\title{
Higher-order Van Hove singularity in magic-angle twisted trilayer graphene
}

\author{
Daniele Guerci $\odot,{ }^{1,2,3}$ Pascal Simon $\odot,{ }^{2}$ and Christophe Mora ${ }^{1,4}$ \\ ${ }^{1}$ Université de Paris, Laboratoire Matériaux et Phénomènes Quantiques, CNRS, F-75013 Paris, France \\ ${ }^{2}$ Université Paris-Saclay, CNRS, Laboratoire de Physique des Solides, 91405 Orsay, France \\ ${ }^{3}$ Center for Computational Quantum Physics, Flatiron Institute, New York, New York 10010, USA \\ ${ }^{4}$ Dahlem Center for Complex Quantum Systems and Fachbereich Physik, Freie Universität Berlin, 14195 Berlin, Germany
}

(Received 8 July 2021; revised 16 January 2022; accepted 21 January 2022; published 9 February 2022)

\begin{abstract}
Twisted trilayer graphene (TTG) has recently emerged experimentally as a fascinating playground to study correlated and exotic superconducting phases. We have found that TTG hosts a zero-energy higher-order Van Hove singularity with an exponent $-1 / 3$ that is stronger than the one predicted in twisted bilayer graphene. This singularity is protected by a threefold rotation symmetry and a combined mirror-particle-hole symmetry and can be tuned with only the twist angle and a perpendicular electric field. It arises from the combined merging of Van Hove singularities and Dirac cones at zero energy, a scheme that goes beyond the recent classifications of Van Hove singularities in single-band models. This structure gives a topological Lifshitz transition, with anomalous exponent $-2 / 5$, which can be achieved in TTG by varying a third control parameter such as the atomic corrugation. The interplay between the nonstandard class of higher-order Van Hove singularities and interaction effects offers an unprecedented platform for studying correlation and superconductivity.
\end{abstract}

DOI: 10.1103/PhysRevResearch.4.L012013

Introduction. Strong correlations generally result from a quenching of electronic motion, comparatively magnifying the strength of electron-electron interactions. This is the case for engineered flat bands, such as for fractional quantum Hall states, but also more generally in proximity to a singularity in the density of states. Tuning the chemical potential at a Van Hove singularity (VHS) [1,2] introduces a large number of single-particle states with negligible energy likely to form a correlated state. Conventional Van Hove singularities entail a logarithmic singularity, but there are also higher-order types $[3,4]$ with more diverging power-law scaling which have been recently classified in single-band electron models [5,6]. Such strong divergence amplifies correlation effects and plays a key role in determining the ordering instabilities in various materials, such as twisted bilayer graphene [4,7-11], biased Bernal stacked bilayer graphene [3], twisted bilayer transition-metal dichalcogenides [12], $\mathrm{Sr}_{3} \mathrm{Ru}_{2} \mathrm{O}_{7}$ [13,14], heavy fermions materials [15], and high- $T_{c}$ superconductors [16]. Moreover, it has recently been shown that the higher-order Van Hove singularity gives rise to a novel non-Fermi-liquid critical state dubbed supermetal [17].

Moiré potentials obtained in graphene multilayer structures by slight misalignment of the stacked layers have proven remarkably fruitful for tuning the single-particle spectrum [18-20] and thereby achieving exotic phases driven by the combined effects of electronic correlation and topology $[21,22]$. Twisted bilayer graphene (TBG) with two rotated

Published by the American Physical Society under the terms of the Creative Commons Attribution 4.0 International license. Further distribution of this work must maintain attribution to the author(s) and the published article's title, journal citation, and DOI. graphene sheets thus exhibits a plethora of interesting phases [23], including correlated symmetry-breaking insulators [24-29], signatures of fragile topology [30,31], orbital magnetism [32-36] and Chern insulators [37-40] with a quantum anomalous Hall effect [41-43], nematicity [44,45], and superconductivity $[22,34,46]$. Significant theoretical progress has also been achieved, especially in understanding the competing nonsuperconducting phases; see, for instance, Refs. [47-64]. A new appealing direction has recently been opened with experiments on twisted trilayer graphene (TTG) [65-80], where only the intercalated layer is rotated by a small angle. Convincing signatures of correlated phases and superconductivity have been observed $[81,82]$, tunable with a perpendicular electrical (displacement) field. Interestingly, data suggest an unconventional superconducting state [83] in the strong-coupling regime of tightly bound pairs and triplet, possibly $p$-wave, pairing.

In this Letter, we argue that a strong higher-order Van Hove singularity emerges in the single-particle spectrum of TTG upon tuning the displacement field and rotation angle. It results from the symmetric merging at zero energy of two standard VHS with opposite energies. Located at the band touching $\mathbf{K}$ point of the moiré Brillouin zone, it falls outside the single-band classification of VHS performed in Refs. [5,6], and also differs from the higher-order VHS identified [4] in TBG. It exhibits the power-law scaling $\omega^{-1 / 3}$, stronger than the $\omega^{-1 / 4}$ predicted for TBG. An even stronger exponent $-2 / 5$ is found by tuning the corrugation, indicating a Lifshitz transition between two topologically incompatible energy contours.

Model and mapping to TBG. The starting point is the continuum model $[18,19]$ for trilayer graphene, where the three layers are stacked with alternating twist angles $\pm \theta$ [65], coupling the three Dirac cones in each valley. It is convenient 

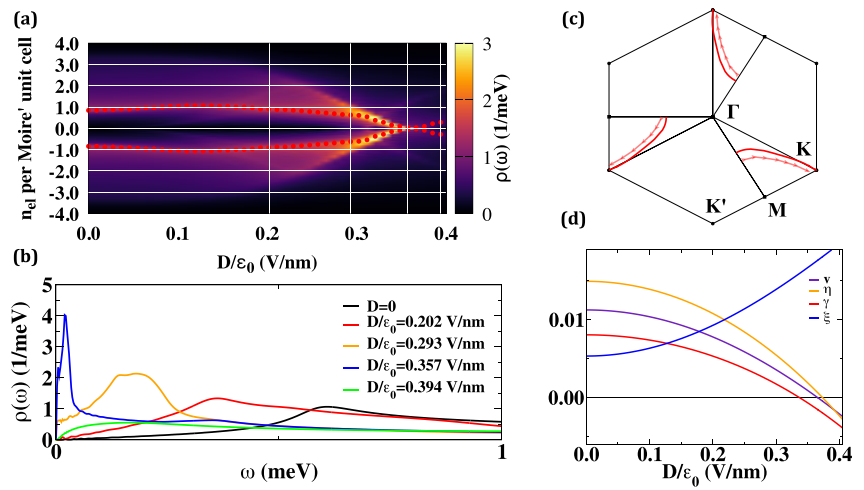

FIG. 1. (a) DOS as a function of the displacement field $D / \epsilon_{0}$ and of the number of electrons per moiré unit cell for $r=0.8$ and $\theta=$ $1.59^{\circ}$. The location of the two VHS is indicated by red dots. (b) Line cuts, corresponding to the white lines in (a), of the DOS as a function of energy for various displacement fields. (c) Evolution as a function of the displacement field of the saddle points (red lines) yielding the strongest peak in the density of states. Arrows indicate increasing $D / \epsilon_{0}$. (d) Values of the parameters of the Hamiltonian (2) $H_{\mathbf{K}}(\mathbf{q})$ as a function of $D / \epsilon_{0}$.

to take advantage of the mirror symmetry $M_{z}$ with respect to the middle layer in the absence of displacement field $D / \varepsilon_{0}$, and write the Hamiltonian in an already layer-rotated basis $[65,68,70,71]$,

$$
H(\mathbf{r})=\left(\begin{array}{ccc}
\hbar v_{0} \boldsymbol{\sigma} \cdot \hat{\mathbf{k}} & \sqrt{2} T(\mathbf{r}) & 0 \\
\sqrt{2} T^{\dagger}(\mathbf{r}) & \hbar v_{0} \boldsymbol{\sigma} \cdot \hat{\mathbf{k}} & U \sigma^{0} / 2 \\
0 & U \sigma^{0} / 2 & \hbar v_{0} \boldsymbol{\sigma} \cdot \hat{\mathbf{k}}
\end{array}\right),
$$

where $\hat{\mathbf{k}}=-i \nabla_{\mathbf{r}}, v_{0}$ is the electron's velocity in graphene, $T(\mathbf{r})=\sum_{j=1}^{3} e^{i \mathbf{q}_{j} \cdot \mathbf{r}} T_{j}$ are the interlayer hoppings with sublattice structure, and $\mathbf{q}_{1}=k_{\theta}(0,1), \mathbf{q}_{2 / 3}=k_{\theta}(\mp \sqrt{3} / 2,-1 / 2)$, where $k_{\theta}=|\mathbf{K}| \theta$ is the distance between the $\mathbf{K}$ points of consecutive layers. $\pm U$ are the gate potentials applied on the top and bottom layers, $U=d D / \varepsilon_{0} \varepsilon$, where $d \simeq 0.3 \mathrm{~nm}$ is the interlayer distance and $\varepsilon_{0}$ is the bare dielectric constant. For simplicity, we neglect screening of the displacement and take $\varepsilon=1$. The interlayer tunneling matrices take the form $T_{j+1}=w_{A A} \sigma^{0}+w_{A B}\left[\sigma^{+} e^{-2 i \pi j / 3}+\sigma^{-} e^{2 i \pi j / 3}\right]$, where $j=0,1,2, w_{A B}=w$ and $w_{A A}=w r$ with $r<1$ due to lattice relaxation effects $[84,85]$, and the $\sigma^{0, \pm}$ matrices act in sublattice space. Hereafter we take $r=0.8$ unless stated otherwise. As Eq. (1) governs the valley $\mathbf{K}$, the valley $\mathbf{K}^{\prime}$ is simply obtained by time-reversal symmetry, i.e., complex conjugation.

At zero displacement $U=0$, Eq. (1) decouples a highvelocity Dirac cone in the odd mirror sector, located at the $\mathbf{K}^{\prime}$ point of the moiré Brillouin zone, from two coupled Dirac cones in the even mirror sector, one at $\mathbf{K}$ and the other one at $\mathbf{K}^{\prime}$. The even sector maps exactly onto the TBG Hamiltonian with a rescaled interlayer tunneling [65], and a corresponding $\sqrt{2}$ enhancement of the magic angle $\theta_{m} \simeq 1.541^{\circ}$ at which the Dirac velocity vanishes. Computing the density of states, we recover the symmetric VHS of TBG in the two active bands [see Figs. 1(a) and 1(b)] observed in STS experiments [86-90]. The VHS is in fact composed of three equivalent saddle points related by $C_{3 z}$ symmetry, i.e., $2 \pi / 3$ rotation in the graphene plane. At a critical angle $\theta_{v} \simeq 1.571^{\circ}$, a higher- order VHS [4] occurs below which each saddle point splits in two. The DOS singularity at $\theta_{v}$ is a power law with exponent $-1 / 4$ and asymmetry ratio $\sim \sqrt{2}$, which convincingly matches the experimentally observed VHS peak [89]. The $C_{2 x}$ symmetry denotes a $\pi$ rotation around $x$ exchanging layer and sublattice indices. It enforces the saddle points to be on the $\boldsymbol{\Gamma M}$ lines of the moiré Brillouin zone for $\theta$ larger than $\theta_{v}$. Throughout the rest of this Letter, we will focus on angles $\theta$ such that the higher-order VHS inherited from TBG does not play a role. Aside from the symmetries $M_{z}, C_{3 z}$, and $C_{2 x}$ already introduced, the model in Eq. (1) is also invariant under the combination $C_{2 z} T$. It anticommutes with the particle-hole symmetry $P$ in the even mirror sector and with $C_{2 x} P$ in both odd and even subspaces.

VHS merging and effective model. We discuss the case of nonzero displacement field $U \neq 0$ and explore the evolution of the density of states at the twist angle $\theta=1.59^{\circ}$. $U$ breaks the mirror symmetry $M_{z}$, the rotation symmetry $C_{2 x}$, and the particle-hole symmetry $P$. However, the product $M_{z} C_{2 x} P$ is preserved [71] together with the remaining symmetries. $M_{z} C_{2 x} P$ acts as anticommuting particle-hole symmetry. It will be very important in stabilizing the new Van Hove singularity; see below. Figure 1(a) shows the density of states as a function of the number of electrons per moiré unit cell and electric displacement field $D / \varepsilon_{0}$. The two particle-hole symmetric VHS, represented as red dots in Fig. 1(a), move towards charge neutrality as $D$ increases and merge at zero energy at a critical $D_{c} / \varepsilon_{0} \simeq 0.37 \mathrm{~V} / \mathrm{nm}$. Introducing the relative dielectric constant $\varepsilon>1$ is going to renormalize $D_{c}$ to larger values of the displacement field. The VHS peaks become concomitantly more pronounced with increasing $D$, as shown in Fig. 1(b), moving towards what seems to be a zero-energy divergence. The corresponding evolution of the $C_{3 z}$ symmetric saddle points with the displacement field is shown in Fig. 1(c). As a consequence of $C_{2 x}$ symmetry breaking, the saddle points leave the $\mathbf{\Gamma M}$ lines and converge towards the $\mathbf{K}$ points in the moiré Brillouin zone. By further increasing $D$ above $D_{c}$, the VHS split again and move away from charge neutrality together with a substantial reduction of the VHS peaks.

In order to gain more analytical insight into the zero-energy merging of VHS and the marked singularity in the density of states, we derive a low-energy approach close to the $\mathbf{K}$ point in the moiré Brillouin zone. Being a high-symmetry point, $\mathbf{K}$ retains some of the symmetries of the model that leaves it invariant: $C_{3 z}, C_{2 z} T$, and $M_{z} C_{2 x} P . C_{2 z} T$ protects a Dirac cone at $\mathbf{K}$ even at the nonzero displacement field. The two degenerate states at $\mathbf{K}$, denoted $u_{\omega}$ and $u_{\omega^{*}}$, can be classified by their $C_{3 z}$ eigenvalues $\omega=e^{2 i \pi / 3}$ and $\omega^{*}$, respectively. $M_{z} C_{2 x} P$ pins these two states at zero energy and, more generally, enforces a fully particle-hole symmetric spectrum at $\mathbf{K}$. The form of the low-energy Hamiltonian in the vicinity of $\mathbf{K}$ is constrained by the symmetries. In the basis of the two degenerate states $\left(u_{\omega}, u_{\omega^{*}}\right)$ defining the Pauli matrices $\tau_{0, x, y, z}$, it takes the $C_{3 z^{-}}$ symmetric form

$$
\begin{aligned}
H_{\mathbf{K}}(\mathbf{q})= & \hbar v \boldsymbol{\tau} \cdot \mathbf{q}+\eta\left(q_{y}^{2}-q_{x}^{2}\right) \tau_{y}-2 \eta q_{x} q_{y} \tau_{x} \\
& +2 \gamma\left(q_{x}^{3}-3 q_{x} q_{y}^{2}\right) \tau_{0}+\xi q^{2} \boldsymbol{\tau} \cdot \mathbf{q},
\end{aligned}
$$

to third order in $\mathbf{q}=\mathbf{k}-\mathbf{K}$, where $q^{2}=q_{x}^{2}+q_{y}^{2}$ and the couplings $\{v, \eta, \gamma, \xi\}$ are calculated by employing a 

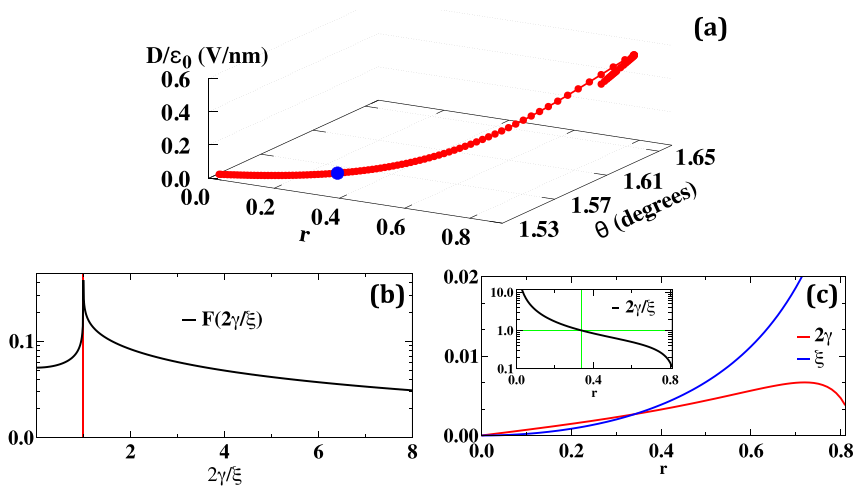

FIG. 2. (a) Three-dimensional trajectory of higher-order VHS originating from the first magic angle $\theta_{C H} \simeq 1.536^{\circ}$ at $r=0$ and $D=0$. The blue dot gives the position of the Lifshitz transition with exponent $-2 / 5$. (b) Evolution of the DOS prefactor in Eq. (4) as a function of $2 \gamma / \xi$. (c) Evolution of $2 \gamma$ and $\xi$ as a function of $r$ along the trajectory of higher-order VHS. The inset shows the ratio $2 \gamma / \xi$ in logarithmic scale.

$\mathbf{k} \cdot \mathbf{p}$ approach with degenerate perturbation theory (see below). The symmetry $M_{z} C_{2 x} P$, acting as $\tau_{x} H_{\mathbf{K}}\left(q_{x}, q_{y}\right) \tau_{x}=$ $-H_{\mathbf{K}}\left(-q_{x}, q_{y}\right)$, forbids other $C_{3 z}$-symmetric terms, such as $q^{2} \tau_{0},\left(q_{x}^{2}-q_{y}^{2}\right) \tau_{x}-2 q_{x} q_{y} \tau_{y}$ or $\left(q_{y}^{3}-3 q_{y} q_{x}^{2}\right) \tau_{0}$, to appear in Eq. (2). The crucial reduction to four coupling constants $\{v, \eta, \gamma, \xi\}$ implies that the higher-order VHS, taking place when $v$ and $\eta$ both vanish, requires the fine tuning of only two parameters of the original model, such as the twist angle $\theta$ and the displacement field $D$.

Beyond the zero-energy subspace $\left(u_{\omega}, u_{\omega^{*}}\right)$, it is convenient to introduce the other eigenstates of Eq. (1) at $\mathbf{K},\left|u_{n}\right\rangle=1$ $\left.u_{n \mathbf{K}}\right\rangle$ with energies $\epsilon_{n}$, and the operator $\mathcal{P}=-\sum_{n}\left|u_{n}\right\rangle\left\langle u_{n}\right|$ $/ \epsilon_{n}$. The values of the coupling constants,

$$
\begin{aligned}
v= & \left\langle u_{\omega^{*}}\left|\sigma^{-}\right| u_{\omega}\right\rangle, \\
\eta= & -\operatorname{Im}\left[\left\langle u_{\omega^{*}}\left|\sigma^{+} \mathcal{P} \sigma^{+}\right| u_{\omega}\right\rangle\right], \\
\gamma= & \operatorname{Re}\left[\left\langle u_{\omega}\left|\sigma^{-} \mathcal{P} \sigma^{-} \mathcal{P} \sigma^{-}\right| u_{\omega}\right\rangle\right], \\
\xi= & \operatorname{Re}\left[\left\langle u_{\omega^{*}}\right| \sigma^{+} \mathcal{P} \sigma^{-} \mathcal{P} \sigma^{-}+\sigma^{-} \mathcal{P} \sigma^{+} \mathcal{P} \sigma^{-}\right. \\
& \left.\quad+\sigma^{-} \mathcal{P} \sigma^{-} \mathcal{P} \sigma^{+}\left|u_{\omega}\right\rangle\right],
\end{aligned}
$$

are calculated from a $\mathbf{k} \cdot \mathbf{p}$ approach at $\mathbf{K}$ (see Supplemental Material [91]) and depend on the details of the spectrum of the trilayer. They are shown in Fig. 1(d) as a function of the displacement field for the angle $\theta=1.59^{\circ}$ measured in [81]. Remarkably, $v$ and $\eta$ vanish for values of $D$ in very close proximity, suggesting the vicinity to the higher-order VHS. It explains the strong feature observed Fig. 1 in the density of states although, rigorously speaking, reaching the higher-order VHS requires the fine tuning of an additional parameter. This can be done by changing the twist angle $\theta$. In order to get a closer look at the exact position of the higherorder VHS, we trace out the values of $D$ and $\theta$ for which the velocity and the curvature of the band dispersion both vanish at the $\mathbf{K}$ point. The result as a function of the corrugation parameter $r$ is shown in Fig. 2(a). As anticipated, we find a line of higher-order VHS in this tridimensional parameter space. We stress again that under the reasonable assumption of a corrugation parameter $r=0.8$ and for the twist angle $\theta=1.59^{\circ}$, one gets very close to the higher-order VHS by simply tuning the electric displacement.

The trajectory of higher-order VHS in Fig. 2(a) originates from a critical angle $\theta_{C H} \simeq 1.536^{\circ}$ where both the displacement field $D$ and the corrugation $r$ are vanishing. This point corresponds in fact to the chiral limit of TBG [92-96], where the whole active band is rigorously flat. At $r=0, \theta=\theta_{C H}$ is also the first magic angle. As $D$ increases, the line of higher-order VHS extends until a critical value of the atomic corrugation $r \simeq 0.842$, above which it is not possible to have $v$ and $\eta$ both vanishing. At smaller twist angles, we find that each magic angle $\theta_{C H}^{(n>1)}$ in the chiral limit $r=0$ is the starting point of a similar line of higher-order VHS.

The higher-order Van Hove singularity. The higher-order VHS appears for a vanishing velocity $v=0$ and curvature $\eta=0$ in Eq. (2). In this case, the dimensional scaling $H_{\mathbf{K}}\left(\lambda^{1 / 3} \mathbf{q}\right)=\lambda H_{\mathbf{K}}(\mathbf{q})$ indicates a power-law scaling,

$$
\rho(\omega)=\xi^{-2 / 3} \mathcal{F}(2 \gamma / \xi)|\omega|^{-1 / 3},
$$

close to charge neutrality, with exponent $-1 / 3$. The Hamiltonian describes a cubic band touching and therefore extends the one-band classification of VHS [5,6]. We would effectively recover one band by setting $\xi=0$ and having $H_{\mathbf{K}}(\mathbf{q})=$ $2 \gamma\left(q_{x}^{3}-3 q_{x} q_{y}^{2}\right) \tau_{0}$. In the vicinity of the higher-order VHS, there are three $C_{3 z}$-symmetric saddle points at positive energy, three at negative, all merging at $\mathbf{K}$ as $v$ and $\eta$ are tuned to zero, as shown in Fig. 1(c), reminiscent of the saddle point merging in the single-band case [3]. In addition, the effective two-band structure entails a pseudospin with a vorticity of +1 at $\mathbf{K}$ protected by $C_{2 z} T$. In the vicinity of the higher-order VHS, $\mathbf{K}$ hosts a +1 Dirac cone surrounded by six additional Dirac cones (see Supplemental Material [91]). The side cones are organized in two $C_{3 z}$-symmetric triplets along $\mathbf{K}^{\prime} \mathbf{K}(\boldsymbol{\Gamma K})$ with vorticity $+1(-1)$. The higher-order VHS occurs precisely as all Dirac cones meet at $\mathbf{K}$, leading to cubic band touching with +1 vorticity. A single Dirac cone at $\mathbf{K}$ remains above the critical value $D>D_{c}$.

In the one-band limit of vanishing $\xi$, the semiclassical orbits are fully open with an anisotropic elliptic umbilic $\left(D_{4}^{-}\right)$structure $[5,6]$ separated by $C_{3 z}$-symmetric lines and a cusp at $\mathbf{q}=0$. Quite the contrary, $\gamma=0$ predicts closed and isotropic semiclassical orbits. The corresponding isoenergy contours are illustrated in Figs. 3(a)-3(c). This difference in topology indicates that a Lifshitz transition is expected to occur as a function of the ratio $2 \gamma / \xi$. This is visible in the divergence in the prefactor $\mathcal{F}$ of Eq. (4) shown in Fig. 2(b). Indeed, for $\xi=2 \gamma$, the spectrum at the higher-order VHS is $E_{ \pm}(\mathbf{q})=\xi q^{3}[ \pm 1+\cos (3 \phi)]$, where $\phi$ is the polar angle between $\mathbf{q}$ and the $x$ axis. It predicts zero-energy lines along the axis at $\phi= \pm \pi / 3, \pi[\pi]$, shown in Fig. 3(b), which are responsible for an even stronger divergence in the density of states. The zero-energy lines are lifted by further expanding the $\mathbf{k} \cdot \mathbf{p}$ approach in Eq. (2) to the first nonvanishing order. With the fourth order being zero, due to the symmetries $C_{3 z}$ and $M_{z} C_{2 x} P$, the dispersion finally takes the form (see Supplemental Material [91])

$$
E_{+}(\mathbf{q})=\xi q^{3}[1+\cos (3 \phi)]+\Lambda q^{5}
$$




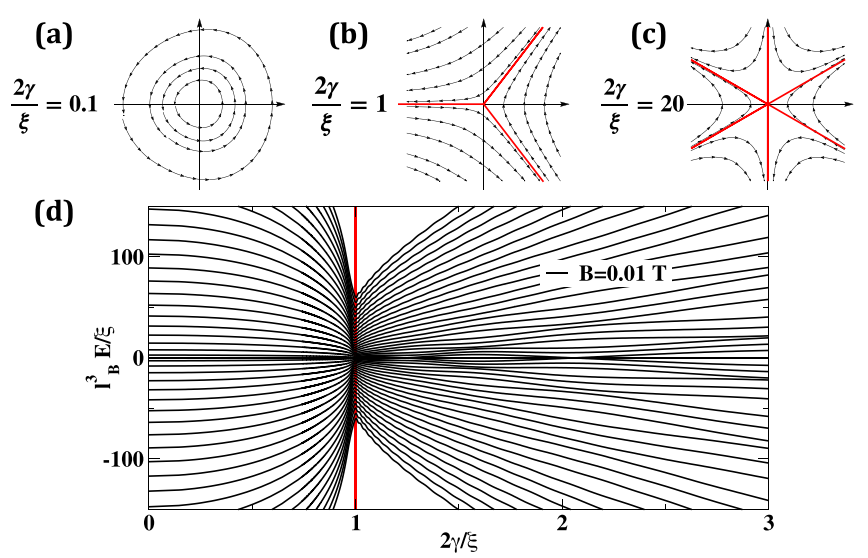

FIG. 3. (a)-(c) The positive isoenergy contours around $\mathbf{K}$ at the higher-order VHS for $2 \gamma / \xi=0.1,2 \gamma / \xi=1.0$ (Lifshitz transition) and $2 \gamma / \xi=20.0$, respectively. Red lines mark the asymptotes of open orbits. (d) Landau level spectrum as a function of $2 \gamma / \xi$ for $B=0.01 \mathrm{~T}$. The vertical red line indicates the location of the Lifshitz transition. The Landau level energy is measured in units of $\xi / l_{B}^{3}$, where $l_{B}$ is the magnetic length $l_{B}=\sqrt{\hbar / e B}$.

in the vicinity of the critical line at $\phi$ (the other lines are inferred by symmetry), where $\Lambda$ is a positive coefficient. Thanks to this term, the divergence in the density of states is cut off at the angle $\delta \phi \sim \sqrt{\Lambda / \xi}(\omega / \Lambda)^{1 / 5}$, where $\delta \phi=\phi-\pi$, and a new power law is obtained,

$$
\rho(\omega) \sim|\omega|^{-2 / 5},
$$

stronger than the naive scaling dimension $-1 / 3$ of the higherorder VHS.

Coming back to the original trilayer model in Eq. (1), the ratio $2 \gamma / \xi$ along the (red) line of higher-order VHS is represented in Fig. 2(c). We then find that the critical point $\xi=2 \gamma$ is reached when the corrugation parameter is $r \simeq 0.34$, corresponding to the twist angle $\theta \simeq 1.543^{\circ}$ and the displacement field $D / \varepsilon_{0} \simeq 0.086 \mathrm{~V} / \mathrm{nm}$. At this point, the DOS critical exponent thus changes to $-2 / 5$, while it is $-1 / 3$ along the rest of the line, indicating a change of topology of the isoenergy contours, from local open orbits when $r<0.34$ to closed ones when $r>0.34$.

The change of Fermi surface topology is reflected in the Landau level (LL) spectrum [97] calculated in Fig. 3(d) for $0.01 \mathrm{~T}$. It is obtained from minimal coupling applied to the effective model in Eq. (2), at vanishing $(v, \eta)$, where the $q^{4}$ terms have been added to regularize the region with open orbits. We find markedly different behavior of both sides of the Lifshitz transition: a regularly spaced structure for closed orbits, corresponding to $2 \gamma / \xi<1$ (and $r>0.34$ ), which evolves into weak oscillations in the regime of open orbits. The LL energy scaling $\propto(n B)^{3 / 2}$ [3] of the higherorder VHS, with LL index $n$ and magnetic field $B$, becomes $\propto(n B)^{5 / 3}$ at the transition due the anomalous $-2 / 5$ DOS exponent. This yields, in Fig. 3(d), a relative collapse of LL at the transition, even more pronounced for decreasing $B$. In addition, the higher-order VHS gives strong corrections to electron self-energy leading to non-Fermi-liquid exponents in the electron conductivity and the specific heat, as discussed in Refs. [13,14].

Conclusions. We derived the existence of a higher-order VHS in mirror-symmetric TTG associated with a strong zero-energy peak in the density of states. In contrast with twisted bilayer graphene, the higher-order VHS (HOVHS) arises from the fusion between the standard finite energy VHS and the Dirac cone at $\mathbf{K}$, and it is protected by $C_{3 z}$ and $M_{z} C_{2 x} P$. As long as these two symmetries are not broken, the HOVHS will still be present with effective parameters which are renormalized by the electron-electron interaction. We emphasize that the peak in the DOS is strongly enhanced in the vicinity of the HOVHS, as shown in Fig. 1(a), and that the position shifts toward charge neutrality. This has two consequences: there will be a larger range of doping for which the Stoner criterion is fulfilled, and this criterion is satisfied for smaller doping to charge neutrality. We argued that current experiments at the magic angle can be brought close to this strong singularity by electric gating, although very strong fields might be needed to compensate screening. This finding has far-reaching consequences, as this strong divergence in the density of states will result in the emergence of a plethora of many-body phenomena that have barely begun to be studied [76]. Moreover, the type of Van Hove singularity that we found differs fundamentally from the standard Van Hove singularity, such as the one classified in Refs. [5,6], as it also involves a Dirac point. This structure gives a topological Lifshitz transition with anomalous exponent $-2 / 5$ that in the regime of small magnetic fields, results in a Landau level energy collapse. Our work opens a horizon in the study of the interplay between new correlated or superconducting phases and exotic Van Hove singularities that are realized in moiré superlattice materials.

Acknowledgments. We have benefited from discussions with K. Kolár and F. von Oppen. This work was supported by the French National Research Agency (Project SIMCIRCUIT, No. ANR-18-CE47-0014-01). The Flatiron Institute is a division of the Simons Foundation.
[1] L. Van Hove, The occurrence of singularities in the elastic frequency distribution of a crystal, Phys. Rev. 89, 1189 (1953).

[2] I. M. Lifshitz, Anomalies of electron characteristics of a metal in the high pressure region, Sov. Phys. JETP 11, 1130 (1960).

[3] A. Shtyk, G. Goldstein, and C. Chamon, Electrons at the monkey saddle: A multicritical Lifshitz point, Phys. Rev. B 95, 035137 (2017).

[4] N. F. Q. Yuan, H. Isobe, and L. Fu, Magic of high-order Van Hove singularity, Nat. Commun. 10, 5769 (2019).
[5] N. F. Q. Yuan and L. Fu, Classification of critical points in energy bands based on topology, scaling, and symmetry, Phys. Rev. B 101, 125120 (2020).

[6] A. Chandrasekaran, A. Shtyk, J. J. Betouras, and C. Chamon, Catastrophe theory classification of fermi surface topological transitions in two dimensions, Phys. Rev. Research 2, 013355 (2020).

[7] Y. Sherkunov and J. J. Betouras, Electronic phases in twisted bilayer graphene at magic angles as a result of Van Hove singularities and interactions, Phys. Rev. B 98, 205151 (2018). 
[8] H. Isobe, N. F. Q. Yuan, and L. Fu, Unconventional Superconductivity and Density Waves in Twisted Bilayer Graphene, Phys. Rev. X 8, 041041 (2018).

[9] Y.-P. Lin and R. M. Nandkishore, Parquet renormalization group analysis of weak-coupling instabilities with multiple high-order Van Hove points inside the Brillouin zone, Phys. Rev. B 102, 245122 (2020).

[10] L. Classen, A. V. Chubukov, C. Honerkamp, and M. M. Scherer, Competing orders at higher-order Van Hove points, Phys. Rev. B 102, 125141 (2020).

[11] D. V. Chichinadze, L. Classen, and A. V. Chubukov, Valley magnetism, nematicity, and density wave orders in twisted bilayer graphene, Phys. Rev. B 102, 125120 (2020).

[12] Z. Bi and L. Fu, Excitonic density wave and spinvalley superfluid in bilayer transition metal dichalcogenide, arXiv: 1911.04493

[13] D. V. Efremov, A. Shtyk, A. W. Rost, C. Chamon, A. P. Mackenzie, and J. J. Betouras, Multicritical Fermi Surface Topological Transitions, Phys. Rev. Lett. 123, 207202 (2019).

[14] C. H. Mousatov, E. Berg, and S. A. Hartnoll, Theory of the strange metal $\mathrm{Sr}_{3} \mathrm{Ru}_{2} \mathrm{O}_{7}$, Proc. Natl. Acad. Sci. USA 117, 2852 (2020).

[15] A. Ramires, P. Coleman, A. H. Nevidomskyy, and A. M. Tsvelik, $\beta$-YBALB 4 : A Critical Nodal Metal, Phys. Rev. Lett. 109, 176404 (2012)

[16] K. Gofron, J. C. Campuzano, A. A. Abrikosov, M. Lindroos, A. Bansil, H. Ding, D. Koelling, and B. Dabrowski, Observation of an "Extended" Van Hove Singularity in $\mathrm{YBa}_{2} \mathrm{Cu}_{4} \mathrm{O}_{8}$ by Ultrahigh Energy Resolution Angle-Resolved Photoemission Phys. Rev. Lett. 73, 3302 (1994).

[17] H. Isobe and L. Fu, Supermetal, Phys. Rev. Research 1, 033206 (2019).

[18] J. M. B. Lopes dos Santos, N. M. R. Peres, and A. H. Castro Neto, Graphene Bilayer with a Twist: Electronic Structure, Phys. Rev. Lett. 99, 256802 (2007).

[19] R. Bistritzer and A. H. MacDonald, Moiré bands in twisted double-layer graphene, Proc. Natl. Acad. Sci. USA 108, 12233 (2011).

[20] G. Trambly de Laissardière, D. Mayou, and L. Magaud, Localization of dirac electrons in rotated graphene bilayers Nano Lett. 10, 804 (2010).

[21] Y. Cao, V. Fatemi, A. Demir, S. Fang, S. L. Tomarken, J. Y. Luo, J. D. Sanchez-Yamagishi, K. Watanabe, T. Taniguchi, E. Kaxiras, R. C. Ashoori, and P. Jarillo-Herrero, Correlated insulator behaviour at half-filling in magic-angle graphene superlattices, Nature (London) 556, 80 (2018).

[22] Y. Cao, V. Fatemi, S. Fang, K. Watanabe, T. Taniguchi, E. Kaxiras, and P. Jarillo-Herrero, Unconventional superconductivity in magic-angle graphene superlattices, Nature (London) 556, 43 (2018).

[23] E. Y. Andrei and A. H. MacDonald, Graphene bilayers with a twist, Nat. Mater. 19, 1265 (2020).

[24] Y. Jiang, X. Lai, K. Watanabe, T. Taniguchi, K. Haule, J. Mao, and E. Y. Andrei, Charge order and broken rotational symmetry in magic-angle twisted bilayer graphene, Nature (London) $\mathbf{5 7 3}$, 91 (2019).

[25] U. Zondiner, A. Rozen, D. Rodan-Legrain, Y. Cao, R. Queiroz, T. Taniguchi, K. Watanabe, Y. Oreg, F. von Oppen, A. Stern et $a l$., Cascade of phase transitions and Dirac revivals in magicangle graphene, Nature (London) 582, 203 (2020).
[26] Y. Saito, J. Ge, L. Rademaker, K. Watanabe, T. Taniguchi, D. A. Abanin, and A. F. Young, Hofstadter subband ferromagnetism and symmetry-broken Chern insulators in twisted bilayer graphene, Nat. Phys. 17, 478 (2021).

[27] J. M. Park, Y. Cao, K. Watanabe, T. Taniguchi, and P. JarilloHerrero, Flavour Hund's coupling, Chern gaps and charge diffusivity in moiré graphene, Nature (London) 592, 43 (2021).

[28] D. Wong, K. P. Nuckolls, M. Oh, B. Lian, Y. Xie, S. Jeon, K. Watanabe, T. Taniguchi, B. A. Bernevig, and A. Yazdani, Cascade of electronic transitions in magic-angle twisted bilayer graphene, Nature (London) 582, 198 (2020).

[29] Y. Xie, B. Lian, B. Jäck, X. Liu, C.-L. Chiu, K. Watanabe, T. Taniguchi, B. A. Bernevig, and A. Yazdani, Spectroscopic signatures of many-body correlations in magic-angle twisted bilayer graphene, Nature (London) 572, 101 (2019).

[30] Z. Song, Z. Wang, W. Shi, G. Li, C. Fang, and B. A. Bernevig, All Magic Angles in Twisted Bilayer Graphene are Topological, Phys. Rev. Lett. 123, 036401 (2019).

[31] H. C. Po, L. Zou, T. Senthil, and A. Vishwanath, Faithful tightbinding models and fragile topology of magic-angle bilayer graphene, Phys. Rev. B 99, 195455 (2019).

[32] J. Liu, Z. Ma, J. Gao, and X. Dai, Quantum Valley Hall Effect, Orbital Magnetism, and Anomalous Hall Effect in Twisted Multilayer Graphene Systems, Phys. Rev. X 9, 031021 (2019).

[33] S.-Y. Li, Y. Zhang, Y.-N. Ren, J. Liu, X. Dai, and L. He, Experimental evidence for orbital magnetic moments generated by moiré-scale current loops in twisted bilayer graphene, Phys. Rev. B 102, 121406(R) (2020).

[34] X. Lu, P. Stepanov, W. Yang, M. Xie, M. A. Aamir, I. Das, C. Urgell, K. Watanabe, T. Taniguchi, G. Zhang et al., Superconductors, orbital magnets and correlated states in magic-angle bilayer graphene, Nature (London) 574, 653 (2019).

[35] C. L. Tschirhart, M. Serlin, H. Polshyn, A. Shragai, Z. Xia, J. Zhu, Y. Zhang, K. Watanabe, T. Taniguchi, M. E. Huber, and A. F. Young, Imaging orbital ferromagnetism in a moiré Chern insulator, arXiv:2006.08053.

[36] D. Guerci, P. Simon, and C. Mora, Moiré lattice effects on the orbital magnetic response of twisted bilayer graphene and Condon instability, Phys. Rev. B 103, 224436 (2021).

[37] Z. Wu, Z. Zhan, and S. Yuan, Lattice relaxation, mirror symmetry and magnetic field effects on ultraflat bands in twisted trilayer graphene, Sci. China Phys. Mech. Astron. 64, 267811 (2021).

[38] Y. Choi, H. Kim, Y. Peng, A. Thomson, C. Lewandowski, R. Polski, Y. Zhang, H. S. Arora, K. Watanabe, T. Taniguchi, J. Alicea, and S. Nadj-Perge, Tracing out correlated Chern insulators in magic angle twisted bilayer graphene, arXiv:2008.11746.

[39] K. P. Nuckolls, M. Oh, D. Wong, B. Lian, K. Watanabe, T. Taniguchi, B. A. Bernevig, and A. Yazdani, Strongly correlated Chern insulators in magic-angle twisted bilayer graphene, Nature (London) 588, 610 (2020).

[40] P. Stepanov, M. Xie, T. Taniguchi, K. Watanabe, X. Lu, A. H. MacDonald, B. A. Bernevig, and D. K. Efetov, Competing Zero-Field Chern Insulators in Superconducting Twisted Bilayer Graphene, Phys. Rev. Lett. 127, 197701 (2021).

[41] A. L. Sharpe, E. J. Fox, A. W. Barnard, J. Finney, K Watanabe, T. Taniguchi, M. A. Kastner, and D. GoldhaberGordon, Emergent ferromagnetism near three-quarters filling in twisted bilayer graphene, Science 365, 605 (2019). 
[42] M. Serlin, C. L. Tschirhart, H. Polshyn, Y. Zhang, J. Zhu, K. Watanabe, T. Taniguchi, L. Balents, and A. F. Young, Intrinsic quantized anomalous Hall effect in a moiré heterostructure, Science 367, 900 (2020).

[43] H. Polshyn, J. Zhu, M. A. Kumar, Y. Zhang, F. Yang, C. L. Tschirhart, M. Serlin, K. Watanabe, T. Taniguchi, A. H. MacDonald et al., Electrical switching of magnetic order in an orbital Chern insulator, Nature (London) 588, 66 (2020).

[44] Y. Choi, J. Kemmer, Y. Peng, A. Thomson, H. Arora, R. Polski, Y. Zhang, H. Ren, J. Alicea, G. Refael et al., Electronic correlations in twisted bilayer graphene near the magic angle, Nat. Phys. 15, 1174 (2019).

[45] Y. Cao, D. Rodan-Legrain, J. M. Park, N. F. Q. Yuan, K. Watanabe, T. Taniguchi, R. M. Fernandes, L. Fu, and P. JarilloHerrero, Nematicity and competing orders in superconducting magic-angle graphene, Science 372, 264 (2021).

[46] M. Yankowitz, S. Chen, H. Polshyn, Y. Zhang, K. Watanabe, T. Taniguchi, D. Graf, A. F. Young, and C. R. Dean, Tuning superconductivity in twisted bilayer graphene, Science $\mathbf{3 6 3}$, 1059 (2019).

[47] Y.-H. Zhang, H. C. Po, and T. Senthil, Landau level degeneracy in twisted bilayer graphene: Role of symmetry breaking, Phys. Rev. B 100, 125104 (2019).

[48] J. Ahn, S. Park, and B.-J. Yang, Failure of Nielsen-Ninomiya Theorem and Fragile Topology in Two-Dimensional systems With Space-time Inversion Symmetry: Application to Twisted Bilayer Graphene at Magic Angle, Phys. Rev. X 9, 021013 (2019).

[49] J. Kang and O. Vafek, Strong Coupling Phases of Partially Filled Twisted Bilayer Graphene Narrow Bands, Phys. Rev. Lett. 122, 246401 (2019).

[50] K. Seo, V. N. Kotov, and B. Uchoa, Ferromagnetic Mott State in Twisted Graphene Bilayers at the Magic Angle, Phys. Rev. Lett. 122, 246402 (2019)

[51] C. Repellin, Z. Dong, Y.-H. Zhang, and T. Senthil, Ferromagnetism in Narrow Bands of Moiré Superlattices, Phys. Rev. Lett. 124, 187601 (2020)

[52] N. Bultinck, S. Chatterjee, and M. P. Zaletel, Mechanism for Anomalous Hall Ferromagnetism in Twisted Bilayer Graphene, Phys. Rev. Lett. 124, 166601 (2020).

[53] R. Pons, A. Mielke, and T. Stauber, Flat-band ferromagnetism in twisted bilayer graphene, Phys. Rev. B 102, 235101 (2020).

[54] Y. Zhang, K. Jiang, Z. Wang, and F. Zhang, Correlated insulating phases of twisted bilayer graphene at commensurate filling fractions: A Hartree-Fock study, Phys. Rev. B 102, 035136 (2020).

[55] J. Liu and X. Dai, Theories for the correlated insulating states and quantum anomalous Hall effect phenomena in twisted bilayer graphene, Phys. Rev. B 103, 035427 (2021).

[56] M. Xie and A. H. MacDonald, Nature of the Correlated Insulator States in Twisted Bilayer Graphene, Phys. Rev. Lett. 124, 097601 (2020).

[57] N. Bultinck, E. Khalaf, S. Liu, S. Chatterjee, A. Vishwanath, and M. P. Zaletel, Ground State and Hidden Symmetry of Magic-Angle Graphene at Even Integer Filling, Phys. Rev. X 10, 031034 (2020).

[58] S. Liu, E. Khalaf, J. Y. Lee, and A. Vishwanath, Nematic topological semimetal and insulator in magic-angle bilayer graphene at charge neutrality, Phys. Rev. Research 3, 013033 (2021).
[59] B. A. Bernevig, Z.-D. Song, N. Regnault, and B. Lian, Twisted bilayer graphene. I. Matrix elements, approximations, perturbation theory, and a $\boldsymbol{k} \cdot \boldsymbol{p}$ two-band model, Phys. Rev. B 103, 205411 (2021).

[60] Z.-D. Song, B. Lian, N. Regnault, and B. A. Bernevig, Twisted bilayer graphene. II. Stable symmetry anomaly, Phys. Rev. B 103, 205412 (2021).

[61] B. A. Bernevig, Z.-D. Song, N. Regnault, and B. Lian, Twisted bilayer graphene. III. Interacting Hamiltonian and exact symmetries, Phys. Rev. B 103, 205413 (2021).

[62] B. Lian, Z.-D. Song, N. Regnault, D. K. Efetov, A. Yazdani, and B. A. Bernevig, Twisted bilayer graphene. IV. Exact insulator ground states and phase diagram, Phys. Rev. B 103, 205414 (2021).

[63] B. A. Bernevig, B. Lian, A. Cowsik, F. Xie, N. Regnault, and Z.-D. Song, Twisted bilayer graphene. V. Exact analytic many-body excitations in Coulomb Hamiltonians: Charge gap, goldstone modes, and absence of cooper pairing, Phys. Rev. B 103, 205415 (2021).

[64] F. Xie, A. Cowsik, Z.-D. Song, B. Lian, B. A. Bernevig, and N Regnault, Twisted bilayer graphene. VI. An exact diagonalization study at nonzero integer filling, Phys. Rev. B 103, 205416 (2021).

[65] E. Khalaf, A. J. Kruchkov, G. Tarnopolsky, and A. Vishwanath, Magic angle hierarchy in twisted graphene multilayers, Phys. Rev. B 100, 085109 (2019).

[66] C. Mora, N. Regnault, and B. A. Bernevig, Flatbands and Perfect Metal in Trilayer Moiré Graphene, Phys. Rev. Lett. 123, 026402 (2019).

[67] X. Li, F. Wu, and A. H. MacDonald, Electronic structure of single-twist trilayer graphene, arXiv:1907.12338.

[68] Z. Zhu, S. Carr, D. Massatt, M. Luskin, and E. Kaxiras, Twisted Trilayer Graphene: A Precisely Tunable Platform for Correlated Electrons, Phys. Rev. Lett. 125, 116404 (2020).

[69] K.-T. Tsai, X. Zhang, Z. Zhu, Y. Luo, S. Carr, M. Luskin, E. Kaxiras, and K. Wang, Correlated insulating states and transport signature of superconductivity in twisted trilayer graphene moiré of moiré superlattices, arXiv:1912.03375.

[70] S. Carr, C. Li, Z. Zhu, E. Kaxiras, S. Sachdev, and A. Kruchkov, Ultraheavy and ultrarelativistic dirac quasiparticles in sandwiched graphenes, Nano Lett. 20, 3030 (2020).

[71] D. Calugaru, F. Xie, Z.-D. Song, B. Lian, N. Regnault, and B. A. Bernevig, Twisted symmetric trilayer graphene: Single-particle and many-body Hamiltonians and hidden nonlocal symmetries of trilayer moiré systems with and without displacement field, Phys. Rev. B 103, 195411 (2021).

[72] J. Shin, B. L. Chittari, and J. Jung, Stacking and gate tunable topological flat bands, gaps and anisotropic strip patterns in twisted trilayer graphene, Phys. Rev. B 104, 045413 (2021).

[73] A. Ramires and J. L. Lado, Emulating Heavy Fermions in Twisted Trilayer Graphene, Phys. Rev. Lett. 127, 026401 (2021).

[74] A. Lopez-Bezanilla and J. L. Lado, Electrical band flattening, valley flux, and superconductivity in twisted trilayer graphene, Phys. Rev. Research 2, 033357 (2020).

[75] C. Lei, L. Linhart, W. Qin, F. Libisch, and A. H MacDonald, Mirror symmetry breaking and lateral stacking shifts in twisted trilayer graphene, Phys. Rev. B 104, 035139 (2021). 
[76] M. Christos, S. Sachdev, and M. S. Scheurer, Correlated insulators, semimetals, and superconductivity in twisted trilayer graphene, arXiv:2106.02063.

[77] E. Lake and T. Senthil, Re-entrant superconductivity through a quantum Lifshitz transition in twisted trilayer graphene, Phys. Rev. B 104, 174505 (2021).

[78] W. Qin and A. H. MacDonald, In-Plane Critical Magnetic Fields in Magic-Angle Twisted Trilayer Graphene, Phys. Rev. Lett. 127, 097001 (2021)

[79] A. Fischer, Z. A. H. Goodwin, A. A. Mostofi, J. Lischner, D. M. Kennes, and L. Klebl, Unconventional superconductivity in magic-angle twisted trilayer graphene, npj Quantum Materials 7, 5 (2022).

[80] Y.-Z. Chou, F. Wu, J. D. Sau, and S. D. Sarma, CorrelationInduced Triplet Pairing Superconductivity in Graphene-Based Moiré Systems, Phys. Rev. Lett. 127, 217001 (2021).

[81] J. M. Park, Y. Cao, K. Watanabe, T. Taniguchi, and P. Jarillo-Herrero, Tunable strongly coupled superconductivity in magic-angle twisted trilayer graphene, Nature (London) $\mathbf{5 9 0}$, 249 (2021).

[82] Z. Hao, A. M. Zimmerman, P. Ledwith, E. Khalaf, D. H. Najafabadi, K. Watanabe, T. Taniguchi, A. Vishwanath, and P. Kim, Electric field-tunable superconductivity in alternatingtwist magic-angle trilayer graphene, Science 371, 1133 (2021).

[83] Y. Cao, J. M. Park, K. Watanabe, T. Taniguchi, and P. Jarillo-Herrero, Large Pauli limit violation and reentrant superconductivity in magic-angle twisted trilayer graphene, arXiv:2103.12083.

[84] N. N. T. Nam and M. Koshino, Lattice relaxation and energy band modulation in twisted bilayer graphene, Phys. Rev. B 96, 075311 (2017).

[85] M. Koshino, N. F. Q. Yuan, T. Koretsune, M. Ochi, K. Kuroki, and L. Fu, Maximally Localized Wannier Orbitals and the Extended Hubbard Model for Twisted Bilayer Graphene, Phys. Rev. X 8, 031087 (2018).

[86] G. Li, A. Luican, J. M. B. Lopes dos Santos, A. H. Castro Neto, A. Reina, J. Kong, and E. Y. Andrei, Observation of Van Hove singularities in twisted graphene layers, Nat. Phys. 6, 109 (2009).

[87] D. Wong, Y. Wang, J. Jung, S. Pezzini, A. M. DaSilva, H.-Z. Tsai, H. S. Jung, R. Khajeh, Y. Kim, J. Lee, S. Kahn,
S. Tollabimazraehno, H. Rasool, K. Watanabe, T. Taniguchi, A. Zettl, S. Adam, A. H. MacDonald, and M. F. Crommie, Local spectroscopy of moiré-induced electronic structure in gate-tunable twisted bilayer graphene, Phys. Rev. B 92, 155409 (2015).

[88] K. Kim, A. DaSilva, S. Huang, B. Fallahazad, S. Larentis, T. Taniguchi, K. Watanabe, B. J. LeRoy, A. H. MacDonald, and E. Tutuc, Tunable moiré bands and strong correlations in smalltwist-angle bilayer graphene, Proc. Natl. Acad. Sci. USA 114, 3364 (2017).

[89] A. Kerelsky, L. J. McGilly, D. M. Kennes, L. Xian, M. Yankowitz, S. Chen, K. Watanabe, T. Taniguchi, J. Hone, C. Dean et al., Maximized electron interactions at the magic angle in twisted bilayer graphene, Nature (London) 572, 95 (2019).

[90] S. Wu, Z. Zhang, K. Watanabe, T. Taniguchi, and E. Y. Andrei, Chern insulators, Van Hove singularities and topological flat bands in magic-angle twisted bilayer graphene, Nat. Mater. 20, 488 (2021).

[91] See Supplemental Material at http://link.aps.org/supplemental/ 10.1103/PhysRevResearch.4.L012013 for details of the calculations.

[92] G. Tarnopolsky, A. J. Kruchkov, and A. Vishwanath, Origin of Magic Angles in Twisted Bilayer Graphene, Phys. Rev. Lett. 122, 106405 (2019).

[93] P. J. Ledwith, G. Tarnopolsky, E. Khalaf, and A. Vishwanath, Fractional Chern insulator states in twisted bilayer graphene: An analytical approach, Phys. Rev. Research 2, 023237 (2020).

[94] S. Becker, M. Embree, J. Wittsten, and M. Zworski, Spectral characterization of magic angles in twisted bilayer graphene, Phys. Rev. B 103, 165113 (2021).

[95] J. Wang, Y. Zheng, A. J. Millis, and J. Cano, Chiral approximation to twisted bilayer graphene: Exact intravalley inversion symmetry, nodal structure, and implications for higher magic angles, Phys. Rev. Research 3, 023155 (2021).

[96] Y. Ren, Q. Gao, A. H. MacDonald, and Q. Niu, WKB Estimate of Bilayer Graphene's Magic Twist Angles, Phys. Rev. Lett. 126, 016404 (2021).

[97] A. Alexandradinata and L. Glazman, Semiclassical theory of Landau levels and magnetic breakdown in topological metals, Phys. Rev. B 97, 144422 (2018). 